E. A. Carter - J. W. MacCluer $\cdot$ B. Dyke $\cdot$

B. V. Howard - R. B. Devereux - S. O. E. Ebbesson •

H. E. Resnick

\title{
Diabetes mellitus and impaired fasting glucose in Alaska Eskimos: the Genetics of Coronary Artery Disease in Alaska Natives (GOCADAN) study
}

Received: 26 July 2005 / Accepted: 19 September 2005 / Published online: 21 December 2005

(C) Springer-Verlag 2005

\begin{abstract}
Aims/hypothesis: We aimed to: (1) define the prevalence of type 2 diabetes and IFG in Eskimos in Norton Sound, Alaska; (2) determine correlates of prevalent diabetes in this population; and (3) compare the prevalence of diabetes in the Genetics of Coronary Artery Disease in Alaska Natives (GOCADAN) Study with other samples of Eskimos, Inuit, American Indians and US blacks, whites and Mexican Americans. Methods: The GOCADAN Study enrolled 1,214 participants $\geq 18$ years who were members of extended pedigrees from the Norton Sound region of Alaska. Diagnosed type 2 diabetes was based on reported use of insulin or hypoglycaemic medications and a medication inventory. Fasting glucose measurements were obtained to ascertain IFG status and undiagnosed diabetes according to American Diabetes Association (ADA) criteria. OGTTs were performed to ascertain diabetes according to the World Health Organization (WHO) definition. We used logistic regression analysis to model factors that were significantly associated with odds of prevalent ADA diabetes. Results: The prevalences of ADA diabetes
\end{abstract}

E. A. Carter · B. V. Howard · S. O. E. Ebbesson ·

H. E. Resnick $(\square)$

Department of Epidemiology and Statistics,

MedStar Research Institute,

6495 New Hampshire Avenue, Suite 201,

Hyattsville, MD 20783, USA

e-mail: helaine.e.resnick@medstar.net

Tel.: +1-301-5607315

Fax: +1-301-5607321

J. W. MacCluer

Southwest Foundation for Biomedical Research,

San Antonio, TX, USA

B. Dyke

Southwest Iconics,

San Antonio, TX, USA

R. B. Devereux

Cornell Medical Center,

New York, NY, USA and IFG were $3.8 \%$ (5.0\% of women; $2.2 \%$ of men) and $15.6 \%$ (13.9\% of women; $17.7 \%$ of men), respectively. In the subset of 787 participants who took the OGTT, the prevalences of ADA and WHO diabetes were 5.1 and $6.9 \%$, respectively. The adjusted odds of ADA diabetes was 2.8 times higher in participants meeting Adult Treatment Panel III criteria for abdominal obesity than in those who did not. The statistically significant sex-related difference in diabetes prevalence did not persist in multivariable analyses. Conclusions/interpretation: Alaska Eskimos have a low prevalence of type 2 diabetes. The high prevalence of IFG indicates that diabetes may become increasingly problematic in this population. Abdominal obesity in women may help explain why diabetes prevalence differs according to sex.

Keywords Alaska Eskimos - Diabetes - Epidemiology · Impaired fasting glucose

Abbreviations ADA: American Diabetes Association . ATP III: Adult Treatment Panel III - GOCADAN: Genetics of Coronary Artery Disease in Alaska Natives .

NHANES: National Health and Nutrition Examination Survey - OR: odds ratio - SHS: Strong Heart Study · WHO: World Health Organization

\section{Introduction}

Until recently, the prevalence of diabetes mellitus and glucose abnormalities has been very low in Alaska Native populations. A 1957 survey of Eskimos identified three cases of diabetes, yielding a prevalence estimate of $0.2 / 1,000$ [1]. Reports from the 1960s show a slight increase in prevalence to $1.4 / 1,000[2,3]$. A review of 1,607 medical records of Alaska Natives from 1979 to 1985 identified nearly 600 Eskimos, Indians and Aleuts who met the 1985 World Health Organization (WHO) criteria for type 2 diabetes [4]. The age-adjusted diabetes prevalence of $15.7 / 1,000$ varied considerably by ethnic group. In a follow-up study conducted from 1986 to 1993, 
the prevalence of diabetes had increased by $22 \%$ to $19.2 / 1,000$. Increases during this period were the most pronounced in the Inupiat Eskimos of the Norton Sound region. The prevalence of WHO-defined diabetes in the Norton Sound Eskimos nearly doubled from 6.7/1,000 in 1985 to $12.6 / 1,000$ in 1993 [5]. Two studies conducted in the $1990 \mathrm{~s}$ estimated the prevalence of WHO diabetes in Eskimos to be between 30.0/1,000 and 66.0/1,000 [6, 7].

The Genetics of Coronary Artery Disease in Alaska Natives (GOCADAN) Study, a longitudinal, populationbased study, recruited 1,214 participants in the Norton Sound region of Alaska. The purposes of this study were: (1) to define the prevalence of IFG and type 2 diabetes by American Diabetes Association (ADA) and WHO definitions; (2) to determine correlates of prevalent ADA diabetes; and (3) to compare the prevalences of diabetes in GOCADAN with other samples of Eskimos, Inuit and American Indians, and US blacks, whites and Mexican Americans.

\section{Subjects, materials and methods}

Study population The study design, survey methods and laboratory techniques of GOCADAN have been published previously in detail [8]. Between October 2000 and April 2004, GOCADAN enrolled 1,214 adult ( $\geq 18$ years) Alaska Eskimos, most of whom belonged to one extended pedigree. Participants were recruited from eight small villages and the town of Nome in the Norton Sound region, an area of 23,000 square miles on the northwest coast of Alaska. Permission was granted by each village council to conduct the study, and written informed consent was obtained from all participants. About 9,000 people live in this region, including 7,700 Eskimos. GOCADAN participants are predominately Inupiat Eskimo (76\%). Other ethnicities in this sample are Yupik Eskimo (8\%), mixed Eskimo (9\%) and other (7\%). Twenty-five participants were not Alaska Natives and were excluded from this report. Overall, $62 \%$ of all eligible family members were recruited. Recruitment in each village was at least $73 \%$ except for one village where recruitment was attempted during the summer hunting and fishing periods when most residents were not available. Examinations at each site consisted of a physical examination, a personal interview, collection of biological specimens and other diagnostic tests.

Definitions and measures of diabetes and IFG To identify undiagnosed diabetes or IFG, participants underwent a blood draw after an overnight ( $12 \mathrm{~h}$ ) fast and were given a standard 2-h 75-g OGTT. Plasma glucose was measured by the hexokinase method on a Hitachi 717 (Roche Diagnostics, Indianapolis, IN, USA). Undiagnosed diabetes was defined by both the 1997 ADA criteria (fasting glucose $\geq 7.0 \mathrm{mmol} / \mathrm{l}$ ) [9] and the $1999 \mathrm{WHO}$ criteria (fasting glucose $\geq 7.0 \mathrm{mmol} / 1$ and/or 2 -h plasma glucose $\geq 7.8$ $\mathrm{mmol} / \mathrm{l})$ [10]. The number of participants who could have been diagnosed according to the ADA definition of type 2 diabetes was 1,056 , the corresponding number for the WHO definition was 787 participants. Fewer participants were potentially diagnosable by the WHO definition because $25 \%$ of the sample did not take the OGTT. IFG was defined in two ways: by the 1997 ADA criteria (6.1$6.9 \mathrm{mmol} / \mathrm{l}$ ) [9] and by the revised 2003 criteria (5.6$6.9 \mathrm{mmol} / \mathrm{l}$ ) [11]. Existing, i.e. previously diagnosed, diabetes was defined as self-reported use of hypoglycaemic agents or insulin, or indication of use of these medications by the medication inventory. As this was based on self-report alone, we were unable to distinguish between type 1 and type 2 diabetes. However, because all participants with diagnosed diabetes were older than 25 years at the time of diagnosis, they were assumed to have type 2 diabetes for the purposes of this report.

Measurement of physical and metabolic characteristics Anthropometry was performed with participants fasting and with an empty bladder. Values taken were rounded to the nearest unit indicated for each measure. Waist circumference was measured with an anthropometric tape applied at the level of the umbilicus with the subject supine. Abdominal obesity was defined using Adult Treatment Panel III (ATP III) criteria [12]: $>102 \mathrm{~cm}$ in men and $>88 \mathrm{~cm}$ in women. Height was measured with a vertical ruler with the participant standing erect on the floor and his/her head in the Frankfort horizontal plane. Weight was measured using a Dectecto balance beam scale. BMI was calculated as weight $(\mathrm{kg}) /$ height $(\mathrm{m})^{2}$ and BMI categories were defined as follows: underweight, $\mathrm{BMI}<18.5$; normal, $18.5 \leq \mathrm{BMI}<25$; overweight, $25 \leq \mathrm{BMI}<30$; obese, $\mathrm{BMI} \geq 30$.

Using a mercury sphygmomanometer (W. A. Baum, Copiague, NY, USA), seated blood pressure was measured three times on the right brachial artery after a 5-min rest period. The mean value of the second and third readings was used as the final measure. Hypertension was defined as systolic blood pressure $\geq 140 \mathrm{mmHg}$, diastolic pressure $\geq 90$ $\mathrm{mmHg}$ or the use of anti-hypertensive medication by selfreport and medication inventory. Participants were categorised as ever-smokers if they reported smoking $>100$ cigarettes in their lifetimes.

Whole blood, plasma, serum and urine were collected from each participant and stored at $-80^{\circ} \mathrm{C}$. Serum lipid samples were analysed using a Vitros 950 (Ortho-Clinical Diagnostics, Rochester, NY, USA) using dry, multilayered, analytical elements coated on a polyester support [13-15]. Fasting plasma insulin levels were measured on a Immulite 2000 analyser using a solid-phase, two-site chemiluminescent assay [16]. $\mathrm{HbA}_{1} \mathrm{c}$ was measured by HPLC. Urine albumin and creatinine were measured from a morning sample. Albuminuria was defined as the ratio of urinary albumin $(\mathrm{mg} / \mathrm{ml})$ to creatinine $(\mathrm{g} / \mathrm{ml})$ of $\geq 30 \mathrm{mg} / \mathrm{g}$.

Statistical methods Chi square and Student's $t$-tests were used to examine differences in proportions and means between categorical and continuous variables, respectively, among those with and without ADA diabetes. Prevalence of diabetes and intermediate glucose abnor- 
Table 1 Prevalence of glucose abnormalities in Alaska Eskimos in GOCADAN, 2000-2003

\begin{tabular}{|c|c|c|c|c|}
\hline & Total & Men & Women & $p^{\mathrm{a}}$ \\
\hline Among entire sample & $n=1056$ & $n=457$ & $n=599$ & \\
\hline Medical history of DM, $n(\%)^{\mathrm{b}}$ & $27(2.6)$ & $6(1.3)$ & $21(3.5)$ & 0.03 \\
\hline ADA-defined DM, $n(\%)^{\mathrm{c}}$ & $40(3.8)$ & $10(2.2)$ & $30(5.0)$ & 0.02 \\
\hline IFG by 1997 ADA definition, $n(\%)^{\mathrm{d}}$ & $40(3.8)$ & $16(3.5)$ & $24(4.0)$ & 0.66 \\
\hline IFG by 2003 ADA definition, $n(\%)^{\mathrm{e}}$ & $164(15.6)$ & $81(17.8)$ & 83 (13.9) & 0.09 \\
\hline Among those with data for OGTT & $n=787$ & $n=334$ & $n=453$ & \\
\hline ADA-defined DM, $n(\%)^{\mathrm{c}}$ & $40(5.1)$ & $10(3.0)$ & $30(6.6)$ & 0.02 \\
\hline WHO-defined DM, $n(\%)$ & $54(6.9)$ & $13(3.9)$ & $41(9.1)$ & $<0.01$ \\
\hline
\end{tabular}

${ }_{\mathrm{a}} p$ value is for difference in proportions by sex

${ }^{\mathrm{b}}$ Use of insulin or hypoglycaemic medications

${ }^{c}$ Fasting glucose $\geq 7.0 \mathrm{mmol} / 1$ or use of insulin or hypoglycaemic medications

${ }^{\mathrm{d}} 1997$ ADA definition of IFG: fasting glucose 6.1-6.9 mmol/1

e2003 ADA definition of IFG: fasting glucose 5.6-6.9 mmol/1

$D M$ type 2 diabetes

malities are reported by sex. Comparisons of WHO diabetes data between GOCADAN and other Eskimo, Inuit and American Indian populations are presented by sex and 10-year age groups. Comparisons of ADA diabetes prevalence between blacks, whites and Mexican American in the 1999-2002 National Health and Nutrition Examination Survey (NHANES) and GOCADAN are limited to those over 20 years of age and are agestandardised using the direct method. Logistic regression analysis was used to identify risk factors that were independently associated with increased odds of prevalent ADA diabetes. Variables were selected for inclusion in the logistic regression model based on a priori knowledge of diabetes risk factors. Multicollinearity diagnostics were performed on the independent variables in the model. Due to the high degree of multicollinearity between BMI and waist circumference, and because of the well-established relationship between central adiposity and type 2 diabetes, we chose to focus on the role of waist circumference in this report.

\section{Results}

Of the 1,189 Alaska Eskimos in the GOCADAN sample, diabetes could not be assessed in 133 participants due to missing fasting glucose measures. Of the 1,056 participants for whom ADA diabetes status could be determined, 40 (3.8\%) had ADA-defined diabetes (Table 1). Of these, 13 (32.5\%; nine women and four men) were undiagnosed at the time of the examination. In the subset of 787 participants with OGTT results, the prevalences of ADA and WHO diabetes were 5.1 and $6.9 \%$, respectively. Although there was no difference in age or sex between participants with and without OGTT data, those missing the OGTT had lower BMI and waist circumference measurements $(p<0.05$, data not shown).

More than one in seven (15.6\%) participants had IFG by the 2003 ADA criteria, a prevalence four times higher than by the 1997 definition (3.8\%). Although the numbers were small in both groups, the prevalences of both ADA and WHO diabetes were more than twice as high in women
Fig. 1 Prevalence of WHO type 2 diabetes by age in Alaska Eskimo, Greenland Inuit, and American Indian men (a) and women (b). Black bars, Alaska Eskimos (GOCADAN) 20002004; open bars, Alaska Eskimos (Alaska-Siberia Project) 1994; hatched bars, Greenland Inuit [18] 1997-2001; grey bars, American Indians (Strong Heart Study) 1989-1992
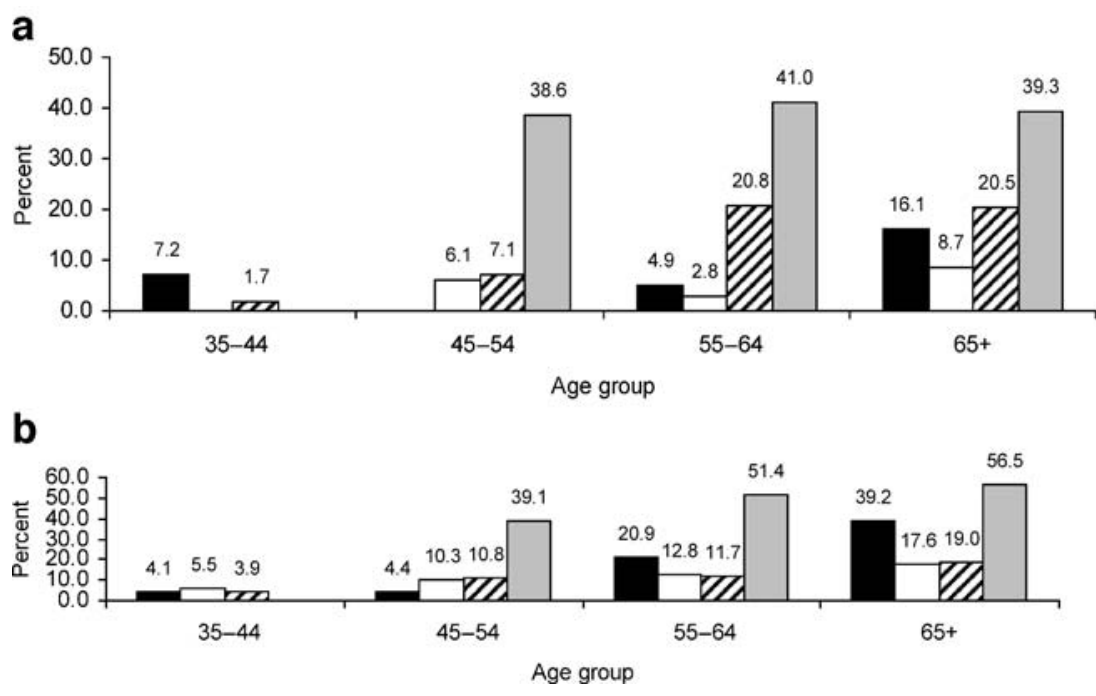


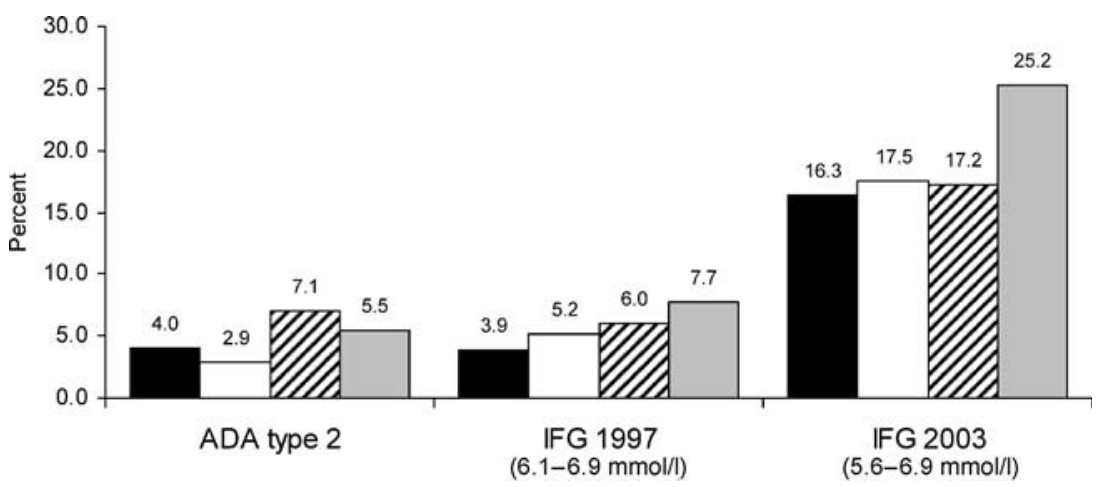

Fig. 2 Age-standardised prevalence of ADA type 2 diabetes and IFG in participants aged 20+ in GOCADAN and NHANES 99-02. Definition of ADA type 2 diabetes in NHANES: fasting glucose $\geq 7.0 \mathrm{mmol} / 1$, self-report of taking insulin or hypoglycaemic agents, or answered yes to the question (other than during pregnancy):

than in men. In contrast, more men than women had IFG by the 2003 definition (17.7 vs 13.9\%), although this result was not statistically significant $(p=0.09)$.

Figure 1 compares the prevalences of WHO diabetes by 10-year age groups in Eskimos in GOCADAN, Eskimos from the Bering Strait region, Greenland Inuit and American Indians. The prevalence of WHO diabetes in Eskimo men in GOCADAN in the older age groups (55$64 ; 65+)$ is higher than among male Eskimos from the Bering Strait region and is lower than the prevalences reported in the Inuit. Among older women there is a higher prevalence of WHO diabetes in GOCADAN than in the other Eskimo or Inuit populations. Both male and female GOCADAN participants had a substantially lower WHO
'Have you ever been told by a doctor or health professional that you have diabetes or sugar diabetes?' Black bars, GOCADAN; open bars, US Whites; hatched bars, US Blacks; grey bars, Mexican Americans

type 2 diabetes prevalence than that of American Indians in the Strong Heart Study (SHS).

Figure 2 compares the age-standardised prevalence of ADA diabetes and IFG among Alaska Eskimos and black, white and Mexican American adults $\geq 20$ years old from NHANES 99-02. Among GOCADAN participants, the prevalence of diabetes was $4.0 \%$, a figure lower than in US blacks (7.1\%) and Mexican Americans (5.5\%) but higher than in US whites $(2.9 \%)$.

Compared with those without ADA diabetes, GOCADAN participants with ADA diabetes were older (59.6 vs 42.0 years), more obese (BMI 32.8 vs $27.4 \mathrm{~kg} / \mathrm{m}^{2}$ ), had a larger waist circumference (101 vs $87 \mathrm{~cm})$ and higher systolic blood pressure (130 vs $119 \mathrm{mmHg}$ ) and triglycer-
Table 2 Characteristics of GOCADAN participants by ADA type 2 diabetes status

DM, type 2 diabetes. Sample size: $n=1056$

${ }^{\mathrm{a} U n d e r w e i g h t: ~} \mathrm{BMI}<18.5$; normal: $18.5 \leq \mathrm{BMI}<25$; overweight: $25 \leq \mathrm{BMI}<30$; obese: $\mathrm{BMI} \geq 30$; data are missing for three participants without DM b $>102 \mathrm{~cm}$ in men; $>88 \mathrm{~cm}$ in women; data available for 39 people with DM and 1,001 without DM

${ }^{\mathrm{c}}$ Systolic blood pressure $\geq 140 \mathrm{mmHg}$ or diastolic blood pressure $\geq 90 \mathrm{mmHg}$ or taking anti-hypertensive medication

${ }^{\mathrm{d}} \mathrm{HbA}_{1} \mathrm{c}>6 \%$

${ }^{\mathrm{e}}$ Data available for 32 people with DM not taking insulin and 1,012 without DM

${ }^{f}$ Albumin:creatinine ratio $>30 \mathrm{mg} / \mathrm{g}$

\begin{tabular}{|c|c|c|c|}
\hline Characteristic & Prevalent ADA DM $(n=40)$ & No ADA DM $(n=1016)$ & $p$ \\
\hline Age (years) & 59.6 & 42.0 & $<0.01$ \\
\hline Female, $n(\%)$ & $30(75)$ & $569(56)$ & 0.02 \\
\hline BMI $\left(\mathrm{kg} / \mathrm{m}^{2}\right)$ & 32.8 & 27.4 & $<0.01$ \\
\hline \multicolumn{4}{|l|}{ BMI category ${ }^{a}$} \\
\hline Underweight & $1(3)$ & $10(1)$ & $<0.01$ \\
\hline Normal & $4(10)$ & $403(40)$ & \\
\hline Overweight & $10(25)$ & $311(31)$ & \\
\hline Obese & $25(63)$ & $289(29)$ & \\
\hline Waist circumference $(\mathrm{cm})$ & 101 & 87 & $<0.01$ \\
\hline ATP III abdominal obesity, $n(\%)^{\mathrm{b}}$ & $29(74)$ & $298(30)$ & $<0.01$ \\
\hline High-school education or more, $n(\%)$ & $21(53)$ & $795(79)$ & $<0.01$ \\
\hline Ever-smoker, $n(\%)$ & $24(60)$ & $835(82)$ & $<0.01$ \\
\hline Systolic blood pressure (mmHg) & 130 & 119 & $<0.01$ \\
\hline Diastolic blood pressure (mmHg) & 75 & 76 & 0.46 \\
\hline Hypertension, $n(\%)^{\mathrm{c}}$ & $32(80)$ & $202(20)$ & $<0.01$ \\
\hline Total cholesterol $(\mathrm{mmol} / \mathrm{l})$ & 5.18 & 5.19 & 0.97 \\
\hline Triglycerides (mmol/l) & 2.45 & 1.40 & 0.02 \\
\hline HDL cholesterol $(\mathrm{mmol} / \mathrm{l})$ & 1.47 & 1.55 & 0.44 \\
\hline LDL cholesterol $(\mathrm{mmol} / \mathrm{l})$ & 2.74 & 3.01 & 0.07 \\
\hline Fibrinogen $(\mathrm{mmol} / \mathrm{l})$ & 11.3 & 9.7 & $<0.01$ \\
\hline Elevated $\mathrm{HbA}_{1} \mathrm{c}, n(\%)^{\mathrm{d}}$ & $30(75)$ & $37(4)$ & $<0.01$ \\
\hline Insulin $(\mathrm{pmol} / \mathrm{l})^{\mathrm{e}}$ & 122 & 58 & $<0.01$ \\
\hline Albuminuria, $n(\%)^{\mathrm{f}}$ & $14(35)$ & $54(5)$ & $<0.01$ \\
\hline
\end{tabular}


Table 3 Logistic regression analysis relating clinical characteristics to prevalent ADA type 2 diabetes, GOCADAN, 2000-2003

\begin{tabular}{llcc}
\hline & Odds ratio & $95 \%$ CI & $p$ \\
\hline Age & 1.04 & $1.01-1.06$ & 0.02 \\
Sex (female vs male) & 1.30 & $0.55-3.63$ & 0.57 \\
ATP III abdominal obesity $^{\mathrm{a}}$ & 2.75 & $1.12-6.77$ & 0.03 \\
Smoking $^{\text {Hypertension }}{ }^{\mathrm{b}}$ & 0.56 & $0.25-1.26$ & 0.16 \\
Triglycerides $^{(\mathrm{mmol} / \mathrm{l})}$ & 5.87 & $2.19-15.78$ & $<0.01$ \\
Albuminuria $^{\mathrm{c}}$ & 1.46 & $1.13-1.88$ & $<0.01$ \\
\hline
\end{tabular}

Sample size: $n=1035$

${ }^{\mathrm{a}}>102 \mathrm{~cm}$ in men; $>88 \mathrm{~cm}$ in women

${ }^{\mathrm{b}}$ Systolic blood pressure $\geq 140 \mathrm{mmHg}$ or diastolic blood pressure $\geq 90 \mathrm{mmHg}$ or taking anti-hypertensive medication

${ }^{c}$ Albumin : creatinine ratio $>30 \mathrm{mg} / \mathrm{g}$ compared with $\leq 30 \mathrm{mg} / \mathrm{g}$

ides ( $2.45 \mathrm{vs} 1.40 \mathrm{mmol} / \mathrm{l})$, and were less likely to have a high-school education (all $p<0.05$ ) (Table 2). Women represent $57 \%$ of participants in this sample but $75 \%$ of those with ADA diabetes. Participants with ADA diabetes had 9.6 times higher odds of albuminuria and 16.1 times higher odds of hypertension than those without ADA diabetes. Total, LDL and HDL cholesterol did not differ significantly between those with and without diabetes. The results did not change when the comparisons were made between those with WHO diabetes and those without.

A logistic regression model was constructed to identify factors that were significantly associated with prevalent ADA diabetes (Table 3). The model is based on 38 participants with ADA diabetes and 997 participants without ADA diabetes with complete data for all covariates. Hypertension (odds ratio [OR] 5.72, 95\% CI 2.12-15.48), albuminuria (OR 3.22, 95\% CI 1.37-5.56) and abdominal obesity (OR 2.75, 95\% CI 1.12-6.77) were significantly associated with prevalent ADA diabetes, as were triglycerides and age. The statistically significant association between sex and diabetes seen in bivariable analyses did not persist in multivariable analyses adjusting for known risk factors and correlates of prevalent diabetes. The Hosmer-Lemeshow goodness-of-fit test suggested that the model fits the data well $(p=0.61)$ and the $c$-statistic indicated that the discriminant ability of the model to correctly identify diabetes cases was $90 \%$ in this sample. The magnitude and direction of the point estimates for these factors were nearly identical when WHO diabetes was examined as the dependent variable (data not shown).

\section{Discussion}

The low prevalence of diabetes in GOCADAN is highly consistent with diabetes prevalence estimates from several previous studies in Alaska Eskimo populations. A 1985 study reported a $4.0 \%$ prevalence of WHO diabetes among Inupiat Eskimos in the Norton Sound region, the same area in which the GOCADAN Study is being conducted [4]. Similarly, the Alaska-Siberia Project, which was conducted in 1994 using protocols very similar to those in GOCADAN, found the prevalence of WHO diabetes in the
Bering Strait region of Alaska to be $2.8 \%$ among Central Yupik, 3.7\% among Inupiat and 9.6\% among Siberian Yupik [6]. All three of these populations are represented in GOCADAN, with $76 \%$ being Inupiat.

The $6.9 \%$ prevalence of WHO diabetes in Eskimos in GOCADAN is about two-thirds that previously reported in the Greenland Inuit $[17,18]$. This result was surprising given the higher mean BMI and greater prevalence of obesity in the GOCADAN population (29.8 vs $19.7 \%$ ). The higher mean age in the Inuit samples did not explain this difference, as age-standardising the results did not appreciably change the diabetes prevalence estimates (data not shown). One possible explanation for this difference is that the definition of diagnosed diabetes in GOCADAN was more restrictive than that in the Greenland study. During the interview participants were asked: 'Has a doctor ever told you that you had diabetes, borderline diabetes or borderline glucose tolerance?'. Because we were unable to distinguish between diabetes and other glucose abnormalities, self-report of diabetes could not be included as part of the definition of diagnosed diabetes, as was the case in the surveys of the Inuit. Relying solely on the use of insulin or hypoglycaemic agents to identify those with diagnosed diabetes possibly resulted in fewer participants being identified as diabetic than if self-report were included.

Although older Alaska Eskimos in the GOCADAN sample appear to have less diabetes relative to the Greenland Inuit, the prevalence of diabetes is still quite low compared with that observed in indigenous populations in other parts of the world. Diabetes prevalence is considerably lower in Eskimos in GOCADAN than in American Indian participants in the SHS. Our data suggest that this difference is explained in large part by lower levels of overweight and abdominal obesity in Eskimos. Mean BMI at the baseline examination of the SHS was $30.8 \mathrm{~kg} / \mathrm{m}^{2}$ and mean waist circumference was $105.2 \mathrm{~cm}(106.7 \mathrm{~cm}$ in women; $102.9 \mathrm{~cm}$ in men). By contrast, in GOCADAN participants aged $45-74$, mean BMI was $27.9 \mathrm{~kg} / \mathrm{m}^{2}$ and mean waist circumference was $88.0 \mathrm{~cm}(88.6 \mathrm{~cm}$ in women; $87.3 \mathrm{~cm}$ in men).

The finding of higher prevalence of diabetes in women is consistent with previous studies in Alaska Eskimos, although this pattern is not seen in Inuit populations 
where diabetes prevalence is higher among men $[17,18]$. Eskimo women have been reported to be 1.6-2.5 times more likely than men to have diabetes $[4,6]$, findings consistent with the twofold higher prevalence of diabetes in GOCADAN women compared with men. A higher prevalence of diabetes in women than men has also been observed in African-American and Hispanic populations [19]. Investigation of the apparent sex-related difference in diabetes in GOCADAN participants suggested that the higher prevalence of abdominal obesity in women $(42 \%)$ compared with men $(11 \%)$ may partly explain the higher prevalence of diabetes in women. Results from logistic regression analyses support this hypothesis. The initial bivariable association between sex and prevalent diabetes was attenuated below statistical significance once diabetes risk factors and correlates such as abdominal obesity, hypertension and albuminuria were added to the model. This result is reported extensively in research in other populations, demonstrating that a centralised distribution of adipose tissue increases risk for type 2 diabetes, even after adjustment for other factors [20-22]. Consistent with these findings, the mean waist circumference among individuals with diabetes was $101 \mathrm{~cm}$ compared with $87 \mathrm{~cm}$ among individuals without diabetes.

Additional sex-stratified analyses revealed that women had higher mean BMI levels (28.6 vs $26.4 \mathrm{~kg} / \mathrm{m}^{2} ; p<0.01$ ) and higher total cholesterol levels than men (5.25 vs $5.09 \mathrm{mmol} / \mathrm{l} ; p<0.01)$ but their LDL levels did not differ. Thus, the sex-related difference in total cholesterol was explained to a large extent by higher HDL levels in women. However, the finding of higher HDL levels in women $(1.64 \mathrm{mmol} / \mathrm{l})$ compared with men $(1.42 \mathrm{mmol} / \mathrm{l})$ was unexpected given the greater frequencies of diabetes and abdominal obesity in women. The sex-related differences in obesity and diabetes cannot be explained at this time. Behavioural factors such as physical activity and diet are likely to be contributory factors but additional research is needed to answer these questions.

An advantage of diabetes prevalence estimates from GOCADAN over earlier estimates in Alaska Natives, which were largely based on registry or patient records data, is that these data are determined from a large, community-based sample. Because our diabetes estimates do not rely on hospital or outpatient records, and because we have a fasting blood draw as well as questionnaire information, we were able to identify 13 cases of undiagnosed as well as 27 cases of previously diagnosed diabetes. The findings of undiagnosed cases of diabetes in GOCADAN is consistent with national estimates as well as with results from both the Alaska-Siberia Project and the 1988 study from Schraer and co-workers that identified one undiagnosed case of diabetes for every diagnosed case of diabetes among Alaska Natives [4, 6, 19]. Therefore the earliest estimates of the extent of diabetes in Native Alaskan populations are likely to be underestimates, perhaps as much as $50 \%$.

It should be noted that clinical practice guidelines and diabetes diagnostic criteria have evolved over time. Since both factors can influence the prevalence estimate of a disease condition, trends in diabetes prevalence over time should be interpreted with caution. Prevalences of both ADA and WHO diabetes were presented so that appropriate comparisons could be made with other studies using either definition. Because OGTTs are not routinely administered in the clinical setting in this and many other populations, ADA diabetes, rather than WHO diabetes, was the focus of the analyses. Thus, the use of the ADA definition is relevant for clinical practice in the Norton Sound region.

There are a number of limitations to this study relating to its cross-sectional design. Because temporal information on exposure and disease are absent, cause and effect relationships between diabetes and its covariates cannot be established. However, some key associations such as those involving fat distribution have been shown in numerous cross-sectional and longitudinal studies. Results from multivariable analyses are consistent with previous research and suggest a relationship between fat distribution and prevalent diabetes in the GOCADAN population. Complications such as hypertension and renal disease were also strong correlates of prevalent diabetes. It is not surprising that, when quantifying associations with prevalent diabetes, the strong effects of diabetes comorbidities overshadow characteristics that may precede the onset of diabetes, particularly abdominal obesity. However, the purpose of this report was to provide a summary of characteristics associated with prevalent diabetes in a large Alaska Eskimo population.

Finally, bias could have been introduced, if the healthiest members of the community had self-selected into the study, while the sickest were unable or unwilling to participate. However the high participation rates in each village suggest that this probably was not the case.

In summary, our data show that although the prevalence of diabetes among Alaska Eskimos is low, nearly $15 \%$ had IFG. Given the current trend toward Westernisation in some historically traditional areas of Alaska, it is likely that diabetes will become a growing public health burden in Eskimo populations. Opportunities may still exist to prevent or delay an epidemic of diabetes in Alaska Eskimos.

Acknowledgements The authors acknowledge the assistance and cooperation of the Eskimo communities of the Norton Sound region, Alaska, without whose support this study would not have been possible. This research is supported by grant U01 HL064244-05 from the National Heart, Lung, and Blood Institute.

\section{References}

1. Scott EM, Griffith IV (1957) Diabetes mellitus in Eskimos. Metabolism 6:320-325

2. Mouratoff GJ, Carroll NV, Scott EM (1967) Diabetes mellitus in Eskimos. JAMA 199:107-112

3. Mouratoff GJ, Scott EM (1973) Diabetes mellitus in Eskimos after a decade. JAMA 226:1345-1346

4. Schraer CD, Lanier AP, Boyko EJ, Gohdes D, Murphy NJ (1988) Prevalence of diabetes mellitus in Alaskan Eskimos, Indians, and Aleuts. Diabetes Care 11:693-700 
5. Schraer CD, Adler AI, Mayer AM, Halderson KR, Trimble BA (1997) Diabetes complications and mortality among Alaska Natives: eight years of observation. Diabetes Care 20:314-321

6. Ebbesson SOK, Schraer CD, Risica PM et al (1998) Diabetes and impaired glucose tolerance in three Alaskan Eskimo populations: the Alaska-Siberia Project. Diabetes Care 21:563-569

7. Burrows NR, Geiss LS, Engelgau MM, Acton KJ (2000) Prevalence of diabetes among Native Americans and Alaska Natives, 1990-1997: an increasing burden. Diabetes Care 23:1786-1790

8. Howard BV, Devereux RB, Cole SA et al (2005) A genetic and epidemiologic study of cardiovascular disease in Alaska Natives (GOCADAN): design and methods. Int J Circumpolar Health 64:206-221

9. The Expert Committee on the Diagnosis and Classification of Diabetes Mellitus (1997) Report of the Expert Committee on the Diagnosis and Classification of Diabetes Mellitus. Diabetes Care 20:1183-1197

10. World Health Organization (1999) Definition, diagnosis and classification of diabetes mellitus and its complications. Part 1: diagnosis and classification of diabetes mellitus. Department of noncommunicable disease surveillance, WHO, Geneva

11. Genuth S, Alberti KG, Bennett P et al (2003) Expert Committee on the Diagnosis and Classification of Diabetes Mellitus. Follow-up report on the diagnosis of diabetes mellitus. Diabetes Care 26:3160-3167

12. National Cholesterol Education Program. Detection, evaluation, and treatment of high blood cholesterol in adults (Adult Treatment Panel III) executive summary. National Institutes of Health. US Dept. of Health and Human Services 2001. NIH Publication No. 01-3670, Bethesda, MD

13. Allain CC, Poon LS, Chan CS, Richmond W, Fu PC (1974) Enzymatic determination of total serum cholesterol. Clin Chem 20:470-475
14. Friedewald WT, Levy RI, Fredrickson DS (1972) Estimation of the concentration of low-density lipoprotein cholesterol in plasma, without use of the preparative ultracentrifuge. Clin Chem 18:499-502

15. Spayd RW, Bruschi B, Burdick BA et al (1978) Multilayer film elements for clinical analysis: applications to representative chemical determinations. Clin Chem 24:1343-1350

16. Burtis CA, Ashwood ER (eds) (1994) Tietz textbook of clinical chemistry. 2nd edn. WB Saunders, Philadelphia, PA, pp 943 944

17. Jorgensen ME, Bjeregaard P, Borch-Johnsen K et al (2002) Diabetes and impaired glucose tolerance among the Inuit population of Greenland. Diabetes Care 25:1766-1771

18. Bjerregaard P, Curtis T, Borch-Johnsen K et al (2003) A population survey of life style and disease in Greenland and among Inuit living in Denmark. Int J Circumpolar Health 62 [Suppl 1]

19. Harris MI, Flegal KM, Cowie CC et al (1998) Prevalence of diabetes, impaired glucose tolerance, and impaired fasting glucose in U.S. adults. The Third National Health and Nutrition Examination Survey, 1988-1994. Diabetes Care 21:518-524

20. Cowie CC, Harris MI, Silverman RE, Johnson EW, Rust KF (1993) Effect of multiple risk factors on differences between blacks and whites in the prevalence of non-insulin-dependent diabetes mellitus in the United States. Am J Epidemiol 137:719-732

21. Haffner SM, Stern MP, Mitchell BD, Hazuda HP, Patterson JK (1990) Incidence of type II diabetes in Mexican Americans predicted by fasting insulin and glucose levels, obesity, and body-fat distribution. Diabetes 39:283-288

22. Ohlson LO, Larsson B, Svardsudd K et al (1985) The influence of body fat distribution on the incidence of diabetes mellitus: 13.5 years of follow-up of the participants in the study of men born in 1913. Diabetes 34:1055-1058 